\title{
Improvement and Enhancement of Natural Gas Hydrate Formation Process by Hummers' Graphene
}

\author{
Ahmad Ghozatloo $^{\text {a,b,* }}$, Mohsen Hosseini ${ }^{\text {b }}$, Mojtaba Shariaty-Niassar ${ }^{\text {b }}$

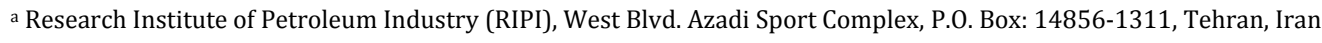 \\ b Transport phenomena and Nanotechnology Laboratory, Department of Chemical Engineering, school of Eng., \\ University of Tehran, P.O. Box: 11155/4563, Tehran, Iran
}

*Corresponding author E-mail: ghozatlooa@ ripi.ir

\begin{abstract}
The effect of graphene in hydrate formation process has been studied for the first time. First, the graphene was synthesized using hummers method and its structure characterization was analyzed by XRD, FTIR and SEM. Graphene nanofluid $1 \%$ wt. was then prepared with no stabilizer or surfactant and was used in natural gas $(92.6 \%$ methane) hydrate formation process with initial pressure of $1000 \mathrm{psig}$ and temperature of $4^{\circ} \mathrm{C}$. Results were compared with deionized water and shown that using small amount of graphene decreased the induction time $61.07 \%$ while increasing the storage capacity by $12.9 \%$. Hummers' graphene (G-Hummers) nanofluid also decreased hydrate dissociation by $41.4 \%$ which causes the hydrates to be more stable. Therefore, G-hummers improved economics of the hydrate formation process which is a hydrate formation technology effective move to industrialization.
\end{abstract}

Keywords: Hydrate, Gas, Graphene, Hummers, Stability, Induction

\section{Introduction}

The order for natural gas carrying and long distances between areas made researchers to find special strategies for handling natural gas transportation. Technologies of gas to wire (GTW), compressed natural gas (CNG), gas to liquid (GTL), liquefied natural gas (LNG) and natural gas hydrates (NGH) are the more industrial among these. Natural gas transportation by hydrates is a new solution in natural gas storage and transportation industry (Thomas et al., 2003).

Hydrate is a crystalline solid consisting of light gas compounds like light hydrocarbons (methane, ethane, propane, etc), $\mathrm{CO}_{2}$ and $\mathrm{N}_{2}$ as guest molecule that are stored in the water molecules cavities (Sun et al., 2003). The role of water molecules in hydrate formation is to form Cage-like vacancies containing relatively large pores by their hydrogen bonds to trap the guest molecules in them (Vangala et al., 2013). Hydrate can have different crystallography structures like cubic structure depending on thermodynamic conditions and size of gas molecules (Nasrifar et al., 2001). These structures include two types of cavities combination. Different structures can be formed by trapping different molecules in these cavities which SI is the most common one (Maghsoodloo et al., 2015). These cavities are unstable without presence of the guest molecule thermodynamically, therefore those molecules that have appropriate size can be trapped in the cavities, form a nonpolar bond with host molecules using Van der Waals forces which resulting a stable structure. This stable structure can be formed in higher temperatures comparing to host molecules freezing point (Fakharian et al., 2012). Four necessary factors (presence of water and gas molecules, high pressure and low temperature) are needed for hydrate formation. Effective factors in hydrate formation divide into operation parameters and promoter parameters using surfactants or nanoparticles. Main operation factors are pressure, temperature, water present amount in the system, mixing speed and volume of the reactor (Verrett et al., 2012). Main effective promoter parameters for nanoparticles are thermal properties, shape, porosity, size, density and stability in base fluids. There have been many researches in order to promote the use of hydrates in gas storage and transportation (Pinnelli et al., 2015), however low rate of formation and stability in structure of hydrate are the two main issues against the industrialization of this process. Hydrate formation is an interfacial phenomenon, so any agent may be able to enhance heat and mass transfer in the interface of water/gas would absolutely enhance rate of hydrate formation and the amount of gas trapped (Takaoki et al., 2002). Therefore, the mentioned issues can be overcome by 
promoting heat and mass transfer phenomena. In recent years, researchers have introduced different promoters for hydrate formation process that most of them were surfactants (Mohammadi et al., 2014). These compounds facilitate the solution of gas in water by reducing the mass transfer resistance, causing more gas molecules to participate in hydrate structure. This will result the increase of storage capacity and reduce the process time. The advantages of these additives are increasing the rate of hydrate formation (Park et al., 2013; Ganji et al., 2007), reducing the formation pressure (Sun et al., 2003; Ganji et al., 2007; Lin et al., 2004) [17-19] and promoting the gas storage capacity (Ganandran et al., 2003; Karaaslan et al., 2002) . The uses of nano particles (Kim et al., 2011; Li et al., 2006; Arjang et al., 2013; Park et al., 2010) like metal oxides and carbon structures have also been common within the last decade. Heat transfer is a very important phenomenon in this process as the gas hydrate formation is exothermic. The heat generated during step of nucleation may destroy the nucleons and resulting decrease in the growth of crystals structures. Gas hydrates are stable in lower temperatures and higher pressures and any parameter disordering this thermodynamic condition causing the hydrates to be destroyed. Therefore the heat generated in the system should be eliminated to maintain the process rate. For this target, an excellent strategy is use of fluids with high thermal conductivity causing enhancement of heat transfer from rector to ambient. Since most nanoparticles (metal and Non-metallic particles) convert the water into high heat transfer coefficient nanofluids (Ghozatloo et al., 2013), using nanofluids instead of base fluid such as water in the reactor, is very effective in accelerating the hydrate formation process. Nanostructures can also increase the mass transfer area because of their nanometric shape and size. Nanostructures can provide more active sites for the nucleation of the hydrate formation process due to their very small sizes. In this study G-hummers is used to improve the hydrate formation process. This nanostructure is not only highly hydrophilic when added to water, but also can improve the water heat transfer coefficient by forming a nanofluid.

\section{Synthesis of G-Hummers}

To synthesis G-hummers method was used. 3gr of graphite powder was first added to $70 \mathrm{ml}$ of concentrated sulfuric acid under temperature of $0^{\circ} \mathrm{C}$ and was stirred for 15 minutes. 9 gr of $\mathrm{KMnO}_{4}$ was then added to the mixture while a circulator was used to keep the temperature under $3^{\circ} \mathrm{C}$ for 60 minutes. The reactor was then transferred to a $40^{\circ} \mathrm{C}$ bath and was completely stirred under that condition for 30 minutes. $150 \mathrm{ml}$ water was then added to the system and the mixture was stirred in ambient temperature. After that, $500 \mathrm{ml}$ water and $15 \mathrm{ml}$ $\mathrm{H}_{2} \mathrm{O}_{2}$ was added under slow stirring until the color of the solution changed to yellow form dark brown. To eliminate metal ions, the solution was washed with $250 \mathrm{ml}$ of $\mathrm{HCl}$. The solution was then washed with water until the $\mathrm{pH}$ reaches 7. Finally, G-hummers separated by filtration and put in oven to be fully dried. Natural gas containing 92.67\% methane in cylinder of pressure 100bar was bought from Gas Product Company and used as feed (table.2). Deionized water was also used in hydrate formation process.

Table 1. composition of natural gas

\begin{tabular}{c|c|c|c|c|c|c|c|c}
\hline Components & $\mathbf{C}_{\mathbf{1}}$ & $\mathbf{C}_{\mathbf{2}}$ & $\mathbf{C}_{\mathbf{3}}$ & $\mathbf{i}-\mathbf{C}_{\mathbf{4}}$ & $\mathbf{n}_{-\mathbf{C}_{\mathbf{4}}}$ & $\mathbf{i}-\mathbf{C}_{\mathbf{5}}$ & $\mathbf{n}_{-} \mathbf{C}_{\mathbf{5}}$ & Other Components \\
\hline \hline$\%$ & 92.67 & 3.13 & 1.08 & 0.351 & 0.224 & 0.213 & 0.119 & 2.213 \\
\hline
\end{tabular}

\subsection{Characterization of G-Hummers}

To study the structure of synthesized G-hummer XRD and FTIR spectroscopy and SEM image are included in the Supplementary information. It is observed (in fig S1) that XRD indicating peak of Ghummers is clearly evident at $2 \theta=11^{\circ}$ (Ghozatloo et al., 2013).

FTIR curve peaks (fig S2) of G-hummers which are clearly apparent at $3410 \mathrm{~cm}^{-1}, 1718 \mathrm{~cm}^{-1}, 1581 \mathrm{~cm}^{-1}$, $1385 \mathrm{~cm}^{-1}, 1218 \mathrm{~cm}^{-1}$ and $1071 \mathrm{~cm}^{-1}$ corresponding to $\mathrm{O}-\mathrm{H}$ stretching of carboxyl groups, $\mathrm{C}=\mathrm{O}$ stretching vibration, stretching of $\mathrm{C}=\mathrm{C}$ bonds, $\mathrm{C}-\mathrm{O}$ stretching vibrations, phenolic $\mathrm{C}-\mathrm{OH}$ stretching and Alkoxy stretching vibration respectively (Shaw et al., 2014; Yaghoubidoust et al., 2014; Tessy et al., 2010; Xinwei et al., 2012). $\mathrm{C}=\mathrm{C}$ bond represents the aromatic (hexagonal) structure of G-Hummers.

It is observed (in fig S3) that the synthesized G-Hummers was consisted of separated folded layers. As this structure can be very well solved in polar solvents, the amount of folding and irregularity of the structure is decreased in solution and SEM image. 


\section{Nanofluid and its use in experimental system of hydrate formation}

In order to set up $1 \%$ wt. G-Hummers nanofluid, $1 \mathrm{gr}$ of G-Hummers add to $99 \mathrm{gr}$ of water and the mixture was put in ultrasonic bath for 10 minutes so that the nanostructure can be fully distributed in water. After that to examine the stability of nanofluid, the amount of sediment was measured after 2 days and after assure about its stability, the nanofluid was used in hydrate formation process.

Hydrate formation process was done in a Parr Steel reactor with a volume of $600 \mathrm{ml}$. To control temperature of the process, the reactor was jacketed and water/ethanol mixture used as coolant fluid in the jacket. It is circulate by a RE-10 Lauda circulator. It is precision is \pm 0.01 degree of centigrade. Two valves are attached to the reactor for inject and exit of the gas. The reactor prepared with a stirrer, which can be adjusted to near 1100 rpm. Fig.1 shows schematic of the experimental system of hydrate formation.

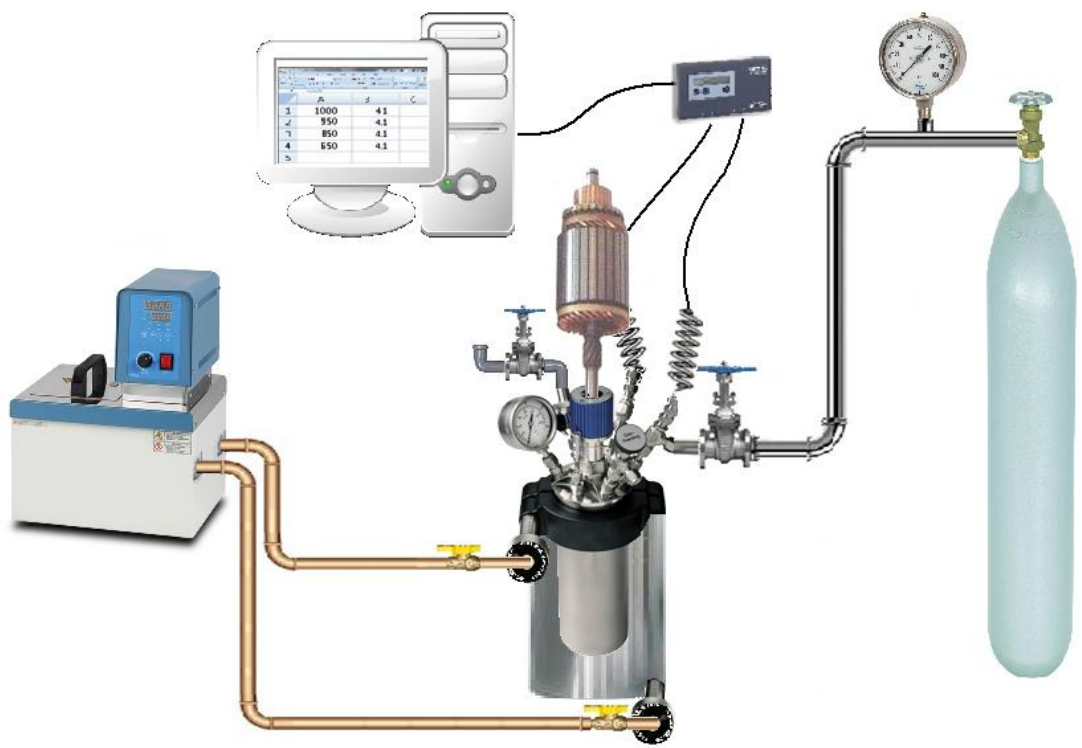

Figure 1. Schematic of the experimental hydrate formation set up

Temperature measurement in the reactor is done with a temperature sensor with a precision of $\pm 0.01 \mathrm{~K}$ equipped with a temperature recording system by electric pulses and is sent to the software. To measure pressure in the reactor a pressure sensor is used in operating range till 140 bars equipped with a pressure recording system by electric pulses with a precision of \pm 0.001 bar. For the experiment, the reactor is perfectly cleaned and dried first. $100 \mathrm{gr}$ of nanofluid was then loaded to the reactor. The reactor is closed and the remaining air in the reactor was removed through the exit valve of the reactor. Then the circulator turned on so that the temperature of the reactor reduced to 4 degree of centigrade. Pressure is also raised to $1000 \mathrm{psig}$. After a while it is observed that there is a small pressure drop in the system because of solution of gas in water. The amount of moles presented in the reactor at any time can be calculated using eq.1.

$\mathrm{n}=\mathrm{PV} / \mathrm{zRT}$

According to eq.1, 1.587 moles natural gas is initially presented inside the reactor. After the first pressure drop, the pressure of the system remains unchanged and the nucleation is performed and the nucleons find their order to form the hydrates. After that a big pressure drop in the system is observed, showing the formation of hydrates. One of the important parameters in study of the hydrate formation process is the storage capacity which is define by the ratio of the gas hydrated volume at standard condition to the hydrate volume. By inserting standard condition values into eq. 1 and using mole number and density fundamental relationships, eq. 2 is generated for calculating hydrate storage capacity.

$\mathrm{V} / \mathrm{V}_{\mathrm{H}}=23.64(\rho . c) / \mathrm{M}_{\mathrm{W}}$

$\rho$ and $\mathrm{M}_{\mathrm{W}}$ in eq.2 are density and molecular weight of hydrate respectively and $\mathrm{c}$ is the mole fraction of the gas trapped in hydrates. The highest capacity of storage in hydrates is 180 volumes by methane (Taheri et al., 2014). After the hydrates formation, the system temperature is reduced to $-10^{\circ} \mathrm{C}$ and the pressure is reduced to atmospheric then and mole percent of hydrate dissociation is measured during 10 hours. 


\section{Results and discussion}

4.1. Hydrate formation in presence of G-Hummers

In order to study the effect of G-Hummers on hydrate formation process, a blank sample consisting of 100gr pure deionized water is used. Based on preliminary tests, which had done in different concentrations of graphene $(0.25,0.50,1.0 \mathrm{wt} \%)$.

Graphene at concentrations of more than $1 \mathrm{wt} \%$ is not completely stable in water and some of it was deposited. Therefore, the maximum authorized concentration of Graphene is reported $1 \mathrm{wt} \%$ for use as nanofluid. The best result had been earned for $1 \mathrm{wt} \%$ among the samples. Summary of The tests were presented in below table.

Table 2. Effect of graphene concentrations on hydrate process

\begin{tabular}{c|c|c|c|c|c|c}
\hline \multirow{2}{*}{$\begin{array}{c}\text { Graphene } \\
(\mathrm{wt} \%)\end{array}$} & \multicolumn{2}{|c|}{ Dissolution time } & \multicolumn{2}{c|}{ Induction time } & \multicolumn{2}{c}{ Process time } \\
\cline { 2 - 6 } & $(\mathrm{min})$ & Reduction (\%) & $(\mathrm{min})$ & Reduction (\%) & (min) & Reduction (\%) \\
\hline \hline 0.0 & 57.0 & 0 & 411.0 & 0 & 604.0 & 0 \\
\hline 0.25 & 52.7 & 7.5 & 354.9 & 13.65 & 592.7 & 1.9 \\
\hline 0.50 & 46.8 & 17.9 & 298.4 & 27.40 & 582.1 & 3.6 \\
\hline 1.0 & 33.4 & 41.4 & 160.0 & 61.07 & 547.5 & 9.4 \\
\hline
\end{tabular}

According to table (2), with increasing of Graphene concentration in water from 0.25 to $1 \mathrm{wt} \%$ the decreasing trend of process time has improved. Then the results of $1 \mathrm{wt} \%$ is the best among other concentration of graphene and reported in this article. Fig. 2 shows the variation of temperature of the reactor over the time, which the rate of heat removal from the system is obvious. According to the natural gas hydrate tests repeatability necessity, each test repeated 4 times and mean was calculated and reported as the results. Figures 2 and 3 were drawn according to the average values of such repeat measurements.

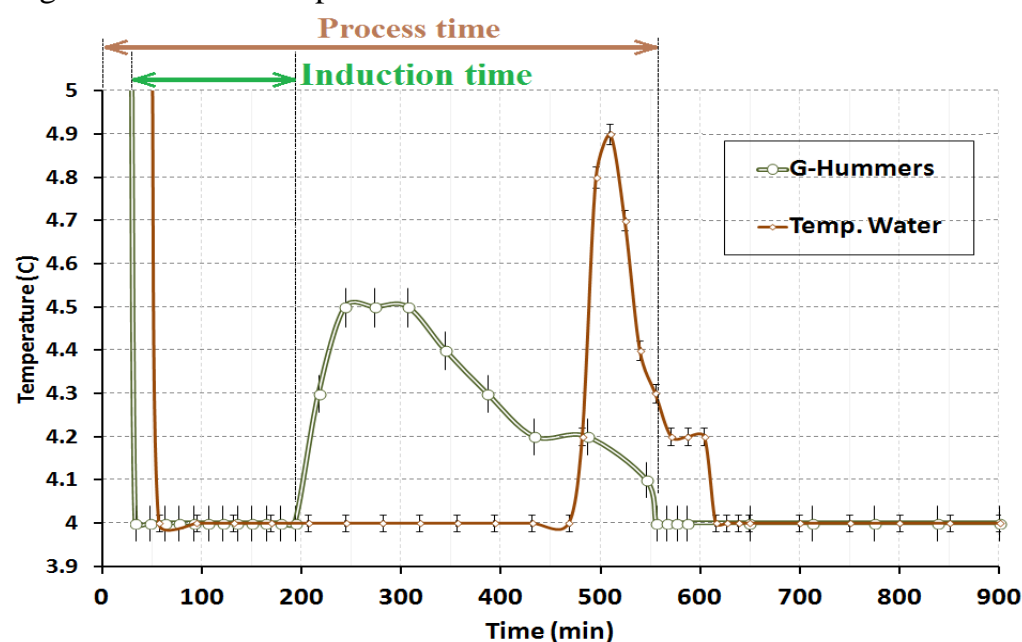

Figure 2. Temperature variations over the time in natural gas hydrate formation process in presence of G-Hummers

As it is observed in fig.2, initial temperature reduction in presence of G-Hummers was faster regarding the heat transfer enhancement of graphene nanofluid. There is also an increase in temperature for both samples during the hydrate formation time. It is also observed that the nanofluid was able to control the temperature, maintaining it more close to $4^{\circ} \mathrm{C}$ and prevent further increase in temperature. Fig. 3 shows pressure-time diagram of the hydrate formation process. According to the repeatability of tests, the error of figures 2 and 3 are less than $1 \%$ and $4.4 \%$ respectively for G-Hummers sample. The error of tests repeatability was calculated using the following equation:

$S D=\delta=\sqrt{\frac{\sum\left(\bar{x}-\mathrm{x}_{\mathrm{i}}\right)^{2}}{n}}$

During the first 33.4 minutes, pressure in the nanofluid sample is dropped to 949.7 psig and remains unchanged for further 160 minutes. This initial pressure drop is due to partial dissolution of the gas in nanofluid and also the initial temperature reduction. The amount of gas dissolved over the first 33.4 minutes was calculated 0.023 moles using eq.1, showing that the dissolution of gas in nanofluid is 23 times more than dissolution in pure water (blank sample) however the dissolution time is decreased by $41.4 \%$. This may be related to the increase in mass transfer area in presence of nanoparticles. Induction time of the process is 160 minutes, which is $41.1 \%$ 
less than the induction time of blank sample. During this period, the dissolved gas begins to nucleation, and these nucleons find their proper order to form hydrates from a supersaturated solution.

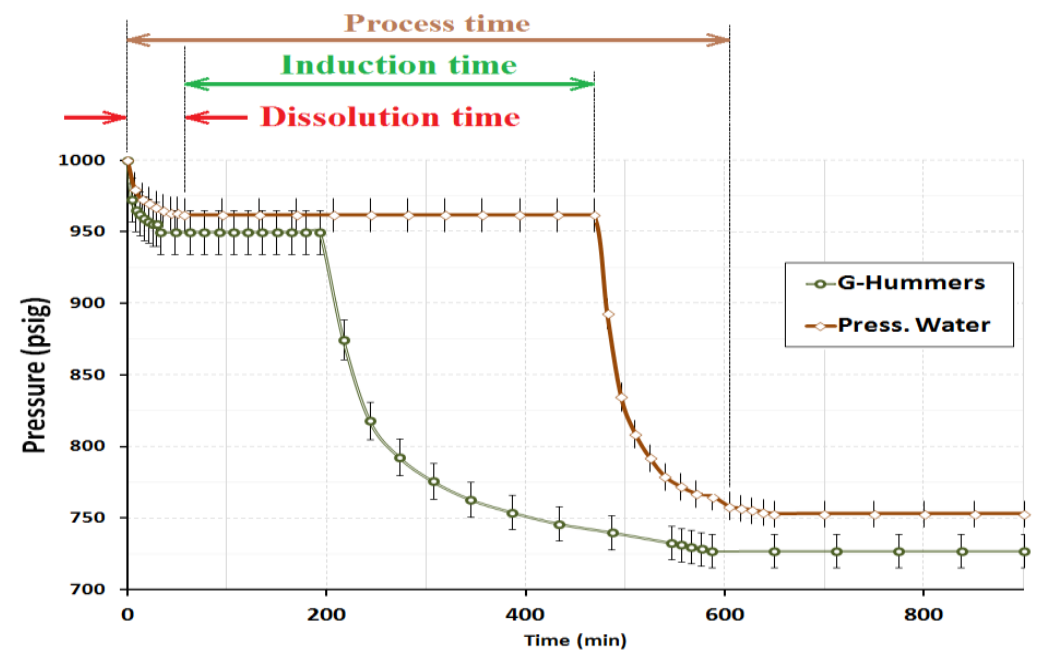

Figure 3. Pressure variations over the time in natural gas hydrate formation process in presence of G-Hummers

After the induction time, a big pressure drop is observed in the system which is due to the formation of hydrates and the pressure of the nanofluid sample and black sample is reduced to $732.7 \mathrm{psig}$ and $758 \mathrm{psig}$ respectively and the pressure does not noticeably change after which shows the competition of the process and thermodynamic equilibrium. Therefore, according to the final thermodynamic state, amount of 0.437 moles natural gas have been trapped in the nanofluid while the corresponding value for the black sample is 0.391 moles.

\subsection{Gas Storage Capacity and Stability of Hydrates}

According to composition of the natural gas (table 1), density and molecular weight of the hydrates calculated $910.4 \mathrm{~kg} / \mathrm{m}^{3}$ and $17.8 \mathrm{gr} / \mathrm{grmole}$ respectively. By substituting these values in eq.2, eq.3 will be generated for calculation of hydrate storage capacity.

$\mathrm{V} / \mathrm{V}_{\mathrm{H}}=1209.2 \mathrm{c}$

It is calculated that the presence of G-Hummers has increased the storage capacity of gas in hydrate by $12.9 \%$ such that the value is raised from 85.3 up to 92.6 . This enhancement is usually because of the increase in the initial dissolved gas in nanofluid. Moreover, G-Hummers has prepared more heterogeneous nucleons so more gas molecules can participate in nucleation process, therefore more hydrates are formed. Using a nanofluid instead of water increase the heat transfer coefficient, resulting acceleration in hydrate formation process and also the amount of the hydrates formed.

To study the stability of hydrates, after the end of the hydrate formation process the temperature of the circulator was adjusted at $-10^{\circ} \mathrm{C}$ for $10 \mathrm{hrs}$ to reach the equilibrium. After that, the remaining gas in the reactor was removed, and the reactor pressure was reduced to atmospheric. During $10 \mathrm{hrs}$, pressure in the reactor recorded. The hydrate dissociation mole percent can be calculated using eq.1. The hydrate dissociation mole percent in presence of G-Hummers and pure water is compared in fig.4. 


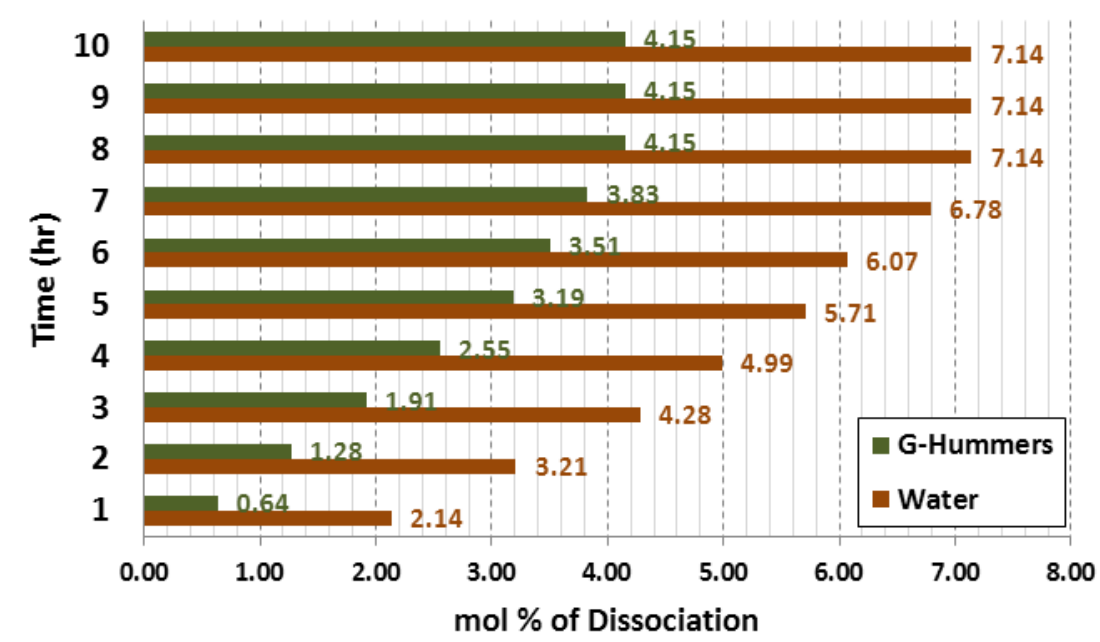

Figure 4. Effect of G-Hummers on rate of dissociation of gas hydrates (Atmospheric pressure/Temerature $-10^{\circ} \mathrm{C}$ )

According to fig.4 during $10 \mathrm{hrs}$, maximum hydrate dissociation in presence of G-Hummers is $4.15 \%$ while the maximum hydrate dissociation for the blank sample is $7.14 \%$ which is $72 \%$ more than the nanofluid sample.

It was observed in the presence of graphene nanofluid, dissociation rate of hydrate is lower than the blank. Therefore, graphene nanosheets have a protective effect on hydrate and they are formed a resistant layer against hydrate dissociation. This phenomenon is reinforced due to graphene nanosheets big network formation in fluid. The network caused to reinforce of hydrate structure in water and so increased the resistance of hydrate to degradation. In other words, the carbon network reinforces structure of hydrate in frame of a strong skeleton structure not only increased storage capacity but also creates long-term stability.

It is also observed that the dissociation in both samples took place in the first 8 hours and after that the hydrates were fully stable. Therefore hydrates formed in presence of G-hummers have a very good stability. This is due to the network formed by dissolution of G-Hummers in water because the carbon network increases the strength of water hydrate structure, resulting of the resistance against dissociation.

\section{Conclusion}

Natural gas hydrate formation process in presence of $1 \%$ wt. G-Hummers nanofluid was studied and observed that G-Hummers can facilitate the heat transfer inside the reactor, causing this process to perform faster, such that the induction time of the process was decreased by $41.4 \%$ while storage capacity in presence of GHummers was increased $12.9 \%$. Furthermore, the stability of the hydrates formed in presence of G-Hummers was increased such that the hydrates in presence of G-Hummers dissociated $42 \%$ less than the hydrates in presence of pure water.

\section{Acknowledgement}

This research was fully financed by the Iran National Science Foundation (INSF), to which the authors are very grateful.

\section{References}

Arjang, S., Manteghian, M., Mohammadi, A., 2013. Effect of synthesized silver nanoparticles in promoting methane hydrate formation at 4.7 MPa and 5.7 MPa, J. Chemical Engineering Research and Design, 91, 6, 1050-1054.

Fakharian, H., Ganji, H., Naderi, Far, A., Kameli, M., 2012. Potato starch as methane hydrate promoter, J. Fuel, 94, 356-360.

Ganji, H., Manteghian, M., Rahimi, M.H., 2007. Effect of mixed compounds on methane hydrate formation and dissociation rates and storage capacity, J. Fuel Processing Technology, 88, 891-895.

Ganji, H., Manteghian, M., Sadaghiani, K., Omidkhah, M.R., Rahimi, M.H., 2007. Effect of different surfactants on methane hydrate formation rate, stability and storage capacity, J. Fuel, 86, 434-441

Ganandran, N.R., 2003. The Effect of Hydrotropes on Gas Hydrates Formation, J. Petrol Sci. Eng., 40, pp.37-46

Ghozatloo, A., Shariaty-Niasar, M., Rashidi, A.M., 2013. Preparation of nanofluids from functionalized graphene by new alkaline method and study on the thermal conductivity and stability, J. International Communications in Heat and Mass Transfer, 42, 89-94. 
Karaaslan, U., Uluneye, E., Parlaktuna, M., 2002. Effect of an Anionic Surfactant on Different Type of Hydrate Structures, J. Petrol. Sci. Eng., 35, 49-57.

Kim, N., Park, S., Kim, H.T., Chun, W., 2011. A comparative study on the enhanced formation of methane hydrate using CM-95 and CM-100, J. International Communications in Heat and Mass Transfer, 38, 31-36.

Li, J., Liang, D., Guo, k., Wang, R., Fan, S., 2006. Formation and dissociation of HFC134a gas hydrate in nano-copper suspension, J. Energy Conversion Management, 47, 201-210.

Lin, W., Chen, G.J., Sun, C.Y., Guo, X.Q., Wu, Z.K., Liang, M.Y., 2004. Effect of Surfactants on The Formation and Dissociation Behavior of Methane Hydrate, J. Chem. Eng. Sci., 59, pp.4449-4455.

Maghsoodloo, B.S., Alamdari, A., 2015. Effect of maize starch on methane hydrate formation/dissociation rates and stability J. Natural Gas Science and Engineering 26, 1-5.

Nasrifar, Kh. Moshfeghian, M., 2001. A model for prediction of gas hydrate formation conditions in aqueous solutions containing electrolytes and/or alcohol, J. Chem Thermodynamics, 33, 999-1014.

Mohammadi, A., Manteghian, M., Haghtalab, A., Mohammadi, A.H., 2014. Rahmati Abkenar M., Kinetic study of carbon dioxide hydrate formation in presence of silver nanoparticles and SDS, J. Chemical Engineering, 237, 387-395.

Park, S., An, E., Lee, S., Chun, W., Kim, N., 2013. Characteristics of methane hydrate formation in carbon nanofluids, J. Industrial and Engineering Chemistry, 18, 443-448.

Park, S., Lee, S., Kim, N., 2010. Effect of multi-walled carbon nanotubes on methane hydrate formation, J. Industrial and Engineering Chemistry, 16, 551-555.

Pinnelli, S.R. Prasad, Vangala, D.C., 2015. Preservation of methane gas in the form of hydrates: Use of mixed hydrates, J. Natural Gas Science and Engineering, 25, 10-14

Shaw, Y.T, Kee, S.L, Siti, K.K, Wan, Ramli W.D., 2014. Graphene production via electrochemical reduction of graphene oxide: Synthesis and characterization, J. Chemical Engineering, 251, 422-434.

Sun, Z.G., Ma, R.S., Wang, R.Z., Guo, K.H., Fan, S.S., 2003. Experimental studying of additives effects on gas storage in hydrate, J. Energy Flues, 17, pp.1180-1185

Sun, Z., Wang, R., Ma, R., Guo, K., Fan, S., 2003. Natural gas storage in hydrate with the presence of promoters, Energy Conv, J. Manag, 44, 2733-2742.

Takaoki, T., Iwasaki, T., Katoh, Y., Arai, T., Horiguchi, K., 2002. Use of Hydrate Pellets for Transformation of Natural GasI; Advantage of Pellet form of Natural Gas Hydrate in Sea Transportation, 4th international Conference on Gas Hydrates, Yokohama.

Taheri, Z., Shabani, M.R., Nazari, K., Mehdizaheh, A., 2014. Natural gas transportation and storage by hydrate technology: Iran case study, J. Natural Gas Science and Engineering, 21, 846-849.

Tessy, T.B, Sundara, R., 2011. Enhanced convective heat transfer using graphene dispersed nanofluids, J. Nanoscale Research letters, 6, 289-298.

Thomas, S., Dawe, R.A., 2003. Review of ways to transport natural gas energy from countries which do not need the gas for domestic use, J. Energy, 28, 1461-1477.

Vangala, D.C, Deepala, Sharma, V.S.G.K., Pinnelli, S.R. Prasad, Sarabu, R.M., 2013. Methane hydrates formation and dissociation in nano silica suspension, J. Natural Gas Science and Engineering, 11, pp.7-11.

Verrett, J., Posteraro, D., 2012. Surfactant effects on methane solubility and mole fraction during hydrate growth, J. Chemical Engineering Science, 84, 80-84.

Xinwei, W., Hongwei, T., Yan, Y., Huan, W., Shumin, W., Weitao, Z., Yichun, L., 2012. Reduced graphene oxide/CdS for efficiently photocatalystic degradation of methylene blue, J. Alloys and Compounds, 524, 5-12.

Yaghoubidoust, F., Dedy, H.B., Sheela, C., Hadi, N., 2014. Effect of graphene oxide on the structural and electrochemical behavior of polypyrrole deposited on cotton fabric, J. Molecular Structure, 1, 486-493. 